\title{
Síndrome de fragilidade em idosos da comunidade: características socio- econômicas e de saúde - um estudo observacional
}

\author{
Frailty in elderly community: socioeconomic and health \\ characteristics - an observational study
}

Mariana C. Buranello ${ }^{1}$, Maycon S. Pegorari², Shamyr S. de Castro³ ${ }^{3}$ Lislei J. Patrizzi ${ }^{4}$

\begin{abstract}
RESUMO
Modelo do estudo: Transversal, observacional e analítico. Objetivo: Verificar a associação da síndrome de fragilidade com as condições socioeconômicas e de saúde em idosos residentes na comunidade. Metodologia: Amostra composta por 54 idosos adscritos à Equipe Saúde da Família do município de Uberaba/MG. A coleta de dados consistiu na avaliação do estado cognitivo, variáveis socioeconômicas, percepção de saúde, morbidades, número de medicamentos e fenótipo de fragilidade, categorizados em não frágil (GN) (nenhum item do fenótipo), pré-frágil (GP) (dois itens do fenótipo) e frágil (GF) (três ou mais itens do fenótipo). Resultados: Constatou-se que $11,1 \%$ dos idosos eram frágeis, 46,3\% pré-frágeis e $42,6 \%$ não frágeis. Não houve diferença significativa para as variáveis socioeconômicas e de saúde entre os grupos, exceto para a maior proporção do número de medicamentos no grupo frágil quando comparado ao pré-frágil e não frágil $(p<0,0028)$. O GF apresentou maior percentual de mulheres, sem escolaridade, viúvos e que moravam com filhos; enquanto que no GP observou-se que a maioria pertencia à faixa etária de 75 anos e mais, 1 a 4 anos de escolaridade e renda de até 1 salário mínimo. Conclusões: O consumo de medicamentos foi maior para os idosos frágeis, indicando a necessidade de atenção especial a essa característica, dado o perfil apresentado. Além disso, os achados da pesquisa ressaltam a relevância dos componentes socioeconômicos e de saúde do idoso em condição de fragilidade.
\end{abstract}

Palavras-chave: Envelhecimento. Idoso Fragilizado. Fragilidade. Nível de Saúde. Fatores Socioeconômicos.

1. Fisioterapeuta. Mestre em Atenção à Saúde. Programa de Pós Graduação Stricto Sensu em Atenção à Saúde da Universidade Federal do Triângulo Mineiro (UFTM). Uberaba, MG/Brasil.

2. Fisioterapeuta. Doutorando em Atenção à Saúde. Programa de Pós-Graduação stricto sensu em Atenção à Saúde da UFTM. Professor Assistente do Curso de Fisioterapia, Universidade Federal do Amapá (UNIFAP).

3. Fisioterapeuta. Doutor em Ciências pela USP-São Paulo. Professor Adjunto do Curso de Fisioterapia da UFTM.

4. Fisioterapeuta. Doutora em Ciências Médicas pela USP-Ribeirão Preto. Professora Adjunta do Curso de Fisioterapia da UFTM.

Correspondencia Universidade Federal do Triângulo Mineiro Curso de Graduação em Fisioterapia Rua Capitão Domingos, 309, Bairro Abadia CEP: 38025-010 Uberaba - MG

Artigo recebido em 05/08/2014 Aprovado para publicação em 02/02/2015 


\begin{abstract}
Study design: Cross-sectional, observational and analytical study. Objective: To investigate the association between frailty syndrome with socioeconomic and health conditions in elderly community residents. Material and Methods: A sample of 54 elderly ascribed to the Family Health Team of Uberaba/MG. Data collection consisted of the evaluation of cognitive status, record of socioeconomic, health perception, morbidities, number of medications and the frailty phenotype, categorized into non-frail (GN) (no items of the phenotype), pre-frail (GP) (two items of the phenotype) and frail (GF) (three or more items of the phenotype). Results: $11.1 \%$ of the group was classified as frail, $46.3 \%$ as pre-frail and $42.6 \%$ as non frail. There was no significant difference for the socioeconomic and health variables between groups. However, the frail group had lower education and income, greater number of morbidities and predominantly female. The use of drugs was statistically higher in the frail group compared to pre-frail and non frail with $100 \%$ of the frail group consuming 3 or more medications. Conclusions: Drug consumption was higher for the frail elderly, indicating the need for special attention to this characteristic, given the different consumption profile shown. Furthermore, the findings of the study emphasize the importance of social components in elderly health impact seen in frailty condition.
\end{abstract}

Keywords: Aging. Frail Elderly; Frailty. Health Status. Socioeconomic Factors.

\section{Introdução}

Fragilidade em idosos pode ser definida como uma síndrome de múltiplas causas e fatores contribuintes, sendo caracterizada pela diminuição da força, endurance muscular e redução da função fisiológica que leva a uma alta vulnerabilidade para desenvolvimento de maior dependência e morte. ${ }^{1}$ Estas alterações refletem-se em condições adversas de saúde como quedas, fraturas, doenças crônicas, hospitalização e mortalidade precoce. ${ }^{2}$

Em relação às condições sociais, culturais e econômicas, o envelhecimento traz maior risco para o desenvolvimento de vulnerabilidades biológicas, socioeconômicas e psicossociais. As características individuais, coletivas e históricas do envelhecimento geram, com diferentes intensidades, as possibilidades para o desenvolvimento de doenças e a dificuldade no acesso aos recursos de proteção social. ${ }^{3} \mathrm{~A}$ identificação desses aspectos, especialmente em idosos frágeis e pré-frágeis poderá favorecer a implementação de ações direcionadas ao cuidado dessa população, tendo em vista o impacto na saúde do idoso, família e sociedade.

As pesquisas nacionais a respeito desta síndrome são recentes, buscando apresentar um perfil da mesma entre os idosos brasileiros, bem como formas de intervenção. Estudo conduzido em sete cidades brasileiras evidenciou prevalência de $9,1 \%$ para a condição de fragilidade e 51,8\% para a pré-fragilidade. Além disso, houve maior percentual de idosos frágeis entre as mulheres, os de 80 anos e mais, os viú- vos, os que nunca foram à escola e os com déficit cognitivo. ${ }^{4}$ Nesta perspectiva, considerando a escassez de estudos no cenário brasileiro, novas investigações a partir do contexto populacional observado são necessárias e a discussão a respeito desta síndrome pode contribuir no sentido de que a saúde dos idosos seja abordada de forma mais abrangente e multifatorial, resultando em benefícios para a população e para o sistema de saúde.

Cabe ressaltar, que no contexto do processo saúde-doença, informações sobre a situação socioeconômica e a condição de fragilidade, são relevantes para os diversos profissionais da área da saúde que desenvolvem intervenções e condutas clínicas junto à população idosa, tendo em vista que os fatores sociais são componentes de grande impacto na saúde dos idosos. ${ }^{5}$ Assim, o objetivo desta pesquisa foi verificar a associação da síndrome de fragilidade com as condições socioeconômicas e de saúde em idosos residentes na comunidade.

\section{Métodos}

Estudo do tipo observacional, analítico e de corte transversal, submetido à apreciação do Comitê de Ética em Pesquisa da Universidade Federal do Triângulo Mineiro (UFTM), Uberaba, MG, Brasil (parecer CEP n $\left.{ }^{\circ} 1815 / 11\right)$ e aprovado, de acordo com as atribuições da Resolução nº 196/96 do Conselho Nacional de Saúde.

Os critérios de inclusão adotados foram idade igual ou superior a 65 anos, de ambos os sexos, ser 
residente permanente no domicílio; ser capaz de deambular, sendo permitido o uso de dispositivo de auxílio para a marcha. Foram excluídos os idosos que apresentaram déficit cognitivo verificado por meio do Mini Exame do Estado Mental (MEEM) com pontos de corte segundo a escolaridade, sendo 28 pontos para escolaridade acima de sete anos, 24 pontos para escolaridade entre 4 e 7 anos, 21 pontos para escolaridade entre 1 e 3 anos e 19 pontos para analfabetos ${ }^{6}$ e doenças neurológicas que impossibilitassem a realização das avaliações.

Foram selecionados 86 participantes adscritos a uma Estratégia de Saúde da Família (ESF) do município de Uberaba - MG, convidados via telefone ou no próprio domicílio a comparecer no local de avaliações, compondo uma amostra de conveniência. Deste total, 11 idosos se recusaram a participar do estudo, um idoso foi a óbito, nove não dispunham de condições físicas para comparecer ao local, cinco mudaram de endereço, seis não atingiram ponto de corte no MEEM, totalizando 32 idosos excluídos. Por fim, a amostra foi constituída por 54 idosos. Os idosos selecionados foram convidados a participar recebendo informações sobre a natureza e objetivos do estudo e, após aceitarem participar da pesquisa, assinaram o termo de consentimento livre e esclarecido. Após a inclusão no estudo, os idosos foram submetidos às entrevistas. A coleta de dados consistiu no registro das variáveis socioeconômicas, antropométricas, indicadores de fragilidade, percepção de saúde, número de morbidades e medicamentos consumidos. Os participantes foram avaliados em um único momento por um examinador devidamente capacitado e treinado.

A mensuração das variáveis sociodemográficas e econômicas ocorreu por meio um questionário estruturado construído especificamente para a pesquisa, constando sexo (masculino e feminino), idade (faixas etárias de $65 \mid-75,75$ e mais), cor da pele (branca, negra e mulata/parda); escolaridade em anos de estudo (sem, $1|-4 ; 4|-8 ; 8$ e mais), renda familiar disposta por faixas de salário (s) mínimo (s) (sem renda, $1|-3,3|-5,5$ e mais), estado conjugal (solteiro/ nunca se casou, casado ou vive com companheiro, viúvo e divorciado/separado), arranjo domiciliar (só, cônjuge/companheiro, outros de sua geração, filhos e netos com ou sem cônjuge). Para os dados clínicos considerou-se: o autorrelato do número de medicamentos utilizados de forma regular nos últimos três meses (até dois, três ou mais) e autorrelato da presen- ça ou ausência de comorbidades, de acordo com a listagem de doenças como: insônia, perda visual, labirintite, hipertensão arterial sistêmica (HAS), doenças cardiovasculares, doença de Chagas, depressão, diabetes, artrose, osteoporose e hérnia de disco.

A percepção de saúde foi avaliada por meio de duas questões, sendo a primeira sobre a percepção do idoso sobre sua saúde de um modo geral e a segunda sobre a sua saúde quando comparada a outros indivíduos da mesma idade. As perguntas foram: "Pensando de um modo geral como o senhor (a) avalia a sua saúde?" (excelente ou muito boa; boa; regular; muito ruim) e "Quando o senhor (a) compara sua saúde com a de outras das pessoas da mesma idade, como o senhor (a) avalia sua saúde no momento atual?" (igual; melhor e pior). ${ }^{7}$

Para a determinação do fenótipo da fragilidade foram seguidos os critérios propostos por Fried et al. ${ }^{2}$ conforme segue 1 - Perda de peso não intencional, avaliada por meio da pergunta "No último ano, o senhor perdeu mais do que $4,5 \mathrm{~kg}$ sem intenção (isto é, sem dieta ou exercício)?". Foi atribuída pontuação positiva para o critério de fragilidade quando houvesse autorrelato de perda superior a $4,5 \mathrm{~kg}$ no último ano ou maior do que 5\% do peso corporal; 2 - Diminuição da força muscular: verificada com base na força de preensão palmar, utilizando o dinamômetro hidráulico manual (NC701/42 - North Coast ${ }^{\mathrm{TM}}$ ), com medida em quilogramas-força (Kgf) e calibrado. Foi considerado o valor médio de três medidas consecutivas com intervalo de 1 minuto de repouso entre estas. Consideraram-se os pontos de corte propostos por Silva et al. ${ }^{8}$, ajustados pelo sexo e IMC; 3 - Auto relato de exaustão: verificada utilizando-se as questões 7 e 20 da versão brasileira da escala de depressão do Center for Epidemiological Studies (CES-D) ${ }^{9} ; 4$ Lentidão na velocidade de marcha: avaliada por meio do tempo de marcha gasto para percorrer uma distância de 4,6 m. Foram adotados os pontos de corte proposto por Silva et al. ${ }^{8}$ ajustados pelo sexo e altura; 5 Baixo nível de atividade física, avaliado pelo Perfil de Atividade Humana (PAH $)^{10}$, considerando o dispêndio semanal de energia (Kcal). Conforme categorização já realizada em outro estudo ${ }^{2}$ foi determinado o percentil 20 dos valores assumidos pelas variáveis na amostra, de acordo com o sexo para o cálculo dos pontos de corte. Pontuaram neste item os idosos que alcançaram gasto calórico por semana menor que $163,23 \mathrm{Kcal}$ para homens e $173,17 \mathrm{Kcal}$ para mulheres. 
Idosos com três ou mais desses itens acima foram classificados como frágeis e aqueles com um ou dois itens, como pré-frágeis. Aqueles com todos os testes negativos para a síndrome da fragilidade foram considerados robustos ou não frágeis. ${ }^{2}$

Os dados foram analisados por meio do programa estatístico InStat, versão 3.05 (GraphPad Software Inc, San Diego, CA, EUA). A análise descritiva foi feita por meio de números brutos, porcentagens, médias e desvio-padrão. O teste de Qui-Quadrado foi usado para testar as diferenças entre as variáveis categóricas e o teste ANOVA one-way para as variáveis numéricas. O nível de significância adotado foi de $5 \%$.

\section{Resultados}

A amostra foi composta por 54 idosos, sendo 32 mulheres $(59,3 \%)$ e 22 homens (40,7\%), com média de idade de $72,9 \pm 6$ anos e índice de massa corpo- ral (IMC) de $26,5 \pm 5,4 \mathrm{Kg} / \mathrm{m}^{2}$. Constituíram-se três grupos por meio da definição operacional proposta por Fried et al. ${ }^{2}$, sendo: Grupo Frágil (GF) (n =6; 11,1\%); Grupo Pré-Frágil (GP) (n=25; 46,3\%); e Grupo Não Frágil (GN) $(n=23 ; 42,6 \%)$. O fluxograma de seleção da amostra bem como a composição dos grupos de acordo com os níveis de fragilidade está expresso na figura 1.

A Tabela 1 apresenta a distribuição das variáveis socioeconômicas entre os grupos. O GF apresentou maior percentual de mulheres, sem escolaridade, viúvos e que moravam com filhos; enquanto que no GP observou-se que a maioria possuía faixa etária de 75 anos e mais, de 1 a 4 anos de escolaridade e renda de até 1 salário mínimo. Constatou-se no GN que a maioria apresentava-se na faixa etária entre 65 a 75 anos, cor da pele branca, com renda de 1 a 3 salários mínimos e eram casados. Não foram observadas diferenças significativas entre os grupos.

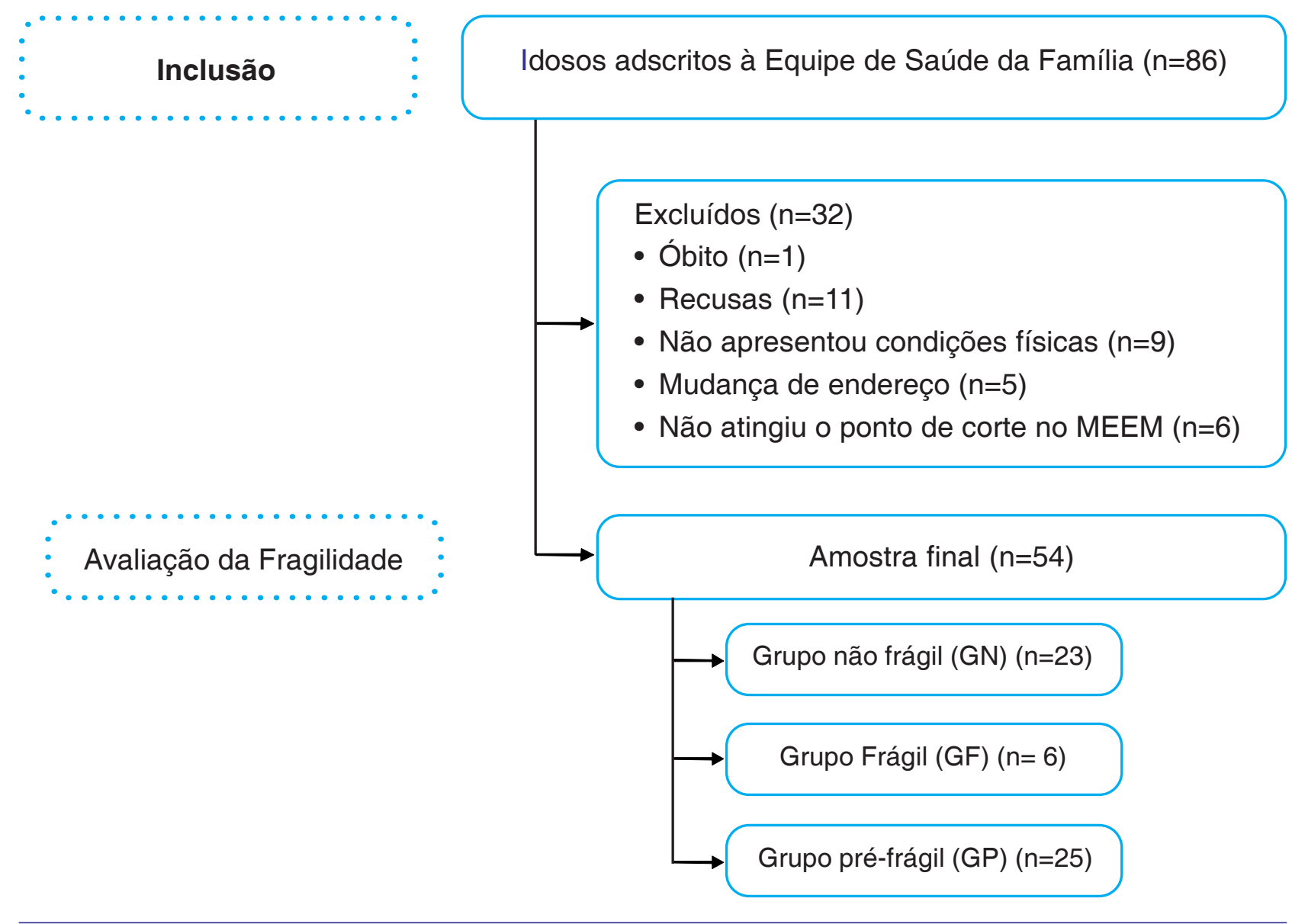

Figura 1. Fluxograma de seleção da amostra. 
Tabela 1: Distribuição das variáveis socioeconômicas dos grupos, Uberaba, MG, Brasil 2011.

\begin{tabular}{|c|c|c|c|c|c|c|c|c|c|}
\hline \multirow[b]{2}{*}{ Variáveis } & \multicolumn{2}{|c|}{ GN } & \multicolumn{2}{|c|}{ GP } & \multicolumn{2}{|c|}{ GF } & \multicolumn{2}{|c|}{ Total } & \multirow[b]{2}{*}{$p^{1}$} \\
\hline & $n(23)$ & $42,6 \%$ & n (25) & $46,3 \%$ & $n(6)$ & $11,1 \%$ & n (54) & $100 \%$ & \\
\hline \multicolumn{10}{|l|}{ Sexo } \\
\hline Feminino & 10 & 43,5 & 17 & 68,0 & 5 & 83,3 & 32 & 59,3 & 0,1000 \\
\hline Masculino & 13 & 56,5 & 8 & 32,0 & 1 & 16,7 & 22 & 40,7 & \\
\hline \multicolumn{10}{|l|}{ Faixa Etária } \\
\hline $65 \mid-75$ & 20 & 86,96 & 16 & 64,0 & 5 & 83,3 & 41 & 75,9 & 0,1610 \\
\hline 75 e mais & 3 & 13,1 & 9 & 36,0 & 1 & 16,97 & 13 & 24,1 & \\
\hline \multicolumn{10}{|l|}{ Escolaridade } \\
\hline Sem & 4 & 17,4 & 5 & 20,0 & 2 & 33,3 & 11 & 20,4 & 0,6920 \\
\hline $1 \mid-4$ & 15 & 65,2 & 19 & 76,0 & 4 & 66,7 & 38 & 70,3 & \\
\hline $4 \mid-8$ & 2 & 8,7 & - & - & - & - & 2 & 3,7 & \\
\hline 8 e mais & 2 & 8,7 & 1 & 4,0 & - & - & 3 & 5,6 & \\
\hline \multicolumn{10}{|l|}{ Cor da pele } \\
\hline Branca & 17 & 73,9 & 17 & 68,0 & 4 & 66,7 & 38 & 70,4 & 0,9800 \\
\hline Preta/Negra & 2 & 8,7 & 3 & 12,0 & 1 & 16,7 & 6 & 11,1 & \\
\hline Mulata/Parda & 4 & 17,4 & 5 & 20,0 & 1 & 16,6 & 10 & 18,5 & \\
\hline \multicolumn{10}{|l|}{ Arranjo domiciliar } \\
\hline Vive Sozinho & 3 & 13,0 & 5 & 20,0 & - & - & 8 & 14,8 & 0,2290 \\
\hline Cônjuge/Companheiro & 12 & 52,2 & 8 & 32,0 & 2 & 33,3 & 22 & 40,7 & \\
\hline Outros de sua geração & 2 & 8,7 & - & - & - & - & 2 & 3,7 & \\
\hline Filhos (com ou sem cônjuge) & 4 & 17,4 & 11 & 44,0 & 4 & 66,7 & 19 & 35,2 & \\
\hline Netos (com ou sem cônjuge) & 2 & 8,7 & 1 & 4,0 & - & - & 3 & 5,6 & \\
\hline \multicolumn{10}{|l|}{ Estado conjugal } \\
\hline Solteiro/nunca se casou & 1 & 4,35 & - & - & - & - & 1 & 1,8 & 0,2550 \\
\hline Casado/Vive com o companheiro & o 16 & 69,6 & 12 & 48,0 & 2 & 33,3 & 30 & 55,6 & \\
\hline Viúvo & 4 & 17,4 & 11 & 44,0 & 4 & 66,7 & 19 & 35,2 & \\
\hline Divorciado/Separado & 2 & 8,7 & 2 & 8,0 & - & - & 4 & 7,4 & \\
\hline \multicolumn{10}{|l|}{ Renda* } \\
\hline Sem renda & 1 & 4,35 & 1 & 4,0 & - & - & 2 & 3,71 & 0,7230 \\
\hline Até 1 salário & 7 & 30,4 & 13 & 52,0 & 3 & 50,0 & 23 & 42,59 & \\
\hline $1 \mid-3$ & 14 & 60,8 & 11 & 44,0 & 3 & 50,0 & 28 & 51,85 & \\
\hline $3 \mid-5$ & - & - & - & - & - & - & - & - & \\
\hline 5 e mais & 1 & 4,35 & - & - & - & - & 1 & 1,85 & \\
\hline
\end{tabular}

GF: grupo frágil; GP: grupo pré-frágil; GN: grupo não frágil. Os dados estão expressos em porcentagem (\%);

*Considerou-se um salário mínimo R $\$ 545,00 ; p^{\text {: }}$ : Diferenças testadas pelo teste de Qui-Quadrado. 
A Tabela 2 apresenta a distribuição das variáveis clínicas e de saúde entre os grupos. Concernente ao uso de medicamentos evidenciou-se maior proporção no GF quando comparado ao GP e GN (p=0,0028). Embora sem diferenças significativas, de modo geral, na percepção de saúde, constatou-se que o maior percentual referiu sua saúde como regular $(50,0 \%)$. Ao comparar sua saúde com a de outras pessoas de mesma idade, a maioria referiu como melhor $(81,48 \%)$.

A Tabela 3 apresenta a distribuição das morbidades autorreferidas entre grupos de idosos. Apesar da ausência significativa entre os grupos, verificouse que entre os pré-frágeis prevaleceram: HAS (80\%), artrose (68\%), doenças cardiovasculares (48\%) e insônia (48\%); enquanto que nos frágeis constatou-se maior percentual de insônia (50\%), HAS (50\%), diabetes $(50 \%)$ e artrose (50\%).

Constatou-se que o GF apresentou maior número de morbidades $(5,83 \pm 2,85)$ em relação ao GP $(5,44 \pm 2,58)$ e GN $(4,39 \pm 2,16)$. Na comparação entre os grupos não foi detectada diferença estatisticamente significativa (GF e GN - $\mathrm{p}=0,6111$; GF e GP $\mathrm{p}=0,9899 ; \mathrm{GN}$ e GP $-\mathrm{p}=0,4310$, ANOVA one-way).

Tabela 2: Distribuição das variáveis clínicas e de saúde dos grupos. Uberaba, MG, Brasil 2011.

\begin{tabular}{|c|c|c|c|c|c|c|c|c|c|}
\hline \multirow[b]{2}{*}{ Variáveis } & \multicolumn{2}{|c|}{ GN } & \multicolumn{2}{|c|}{ GP } & \multicolumn{2}{|c|}{ GF } & \multicolumn{2}{|c|}{ Total } & \multirow[b]{2}{*}{$p^{1}$} \\
\hline & $n(23)$ & $42,6 \%$ & $n(25)$ & $46,3 \%$ & $n(6)$ & $11,1 \%$ & $\mathrm{n}(54)$ & $100 \%$ & \\
\hline \multicolumn{10}{|l|}{ Uso de medicamentos } \\
\hline Até 2 & 9 & 36,0 & 1 & 4,0 & - & - & 10 & 18,5 & 0,0028 \\
\hline 3 ou mais & 14 & 63,4 & 24 & 96,0 & 6 & 100,0 & 44 & 81,5 & \\
\hline \multicolumn{10}{|l|}{ Percepção de saúde } \\
\hline Excelente ou muito boa & 4 & 17,39 & 4 & 16,0 & 2 & 33,3 & 10 & 18,5 & 0,3690 \\
\hline Boa & 8 & 34,78 & 7 & 28,0 & - & - & 15 & 27,8 & \\
\hline Regular & 11 & 47,83 & 13 & 52,0 & 3 & 50,0 & 27 & 50,0 & \\
\hline Muito Ruim (ruim ou má) & - & - & 1 & 4,0 & 1 & 16,6 & 2 & 3,7 & \\
\hline \multicolumn{10}{|c|}{ Saúde comparada a de outras pessoas da mesma idade } \\
\hline Igual & 4 & 17,39 & 2 & 8,00 & 3 & 50,00 & 9 & 16,7 & 0,1080 \\
\hline Melhor & 18 & 78,26 & 23 & 92,00 & 3 & 50,00 & 44 & 81,5 & \\
\hline Pior & 1 & 4,35 & - & - & - & - & 1 & 1,8 & \\
\hline
\end{tabular}

GF: grupo frágil; GP: grupo pré-frágil; GN: grupo não frágil; IMC: índice de massa corporal.

Os dados estão expressos em porcentagem (\%); ${ }^{\star} p<0,05 ; p^{2}$ : Diferenças testadas pelo teste de Qui-Quadrado.

Tabela 3: Distribuição das morbidades autorreferidas dos grupos. Uberaba, MG, Brasil 2011.

\begin{tabular}{|c|c|c|c|c|c|c|c|c|c|}
\hline \multirow[b]{2}{*}{ Variáveis } & \multicolumn{2}{|c|}{ GN } & \multicolumn{2}{|c|}{ GP } & \multicolumn{2}{|c|}{ GF } & \multicolumn{2}{|c|}{ Total } & \multirow[b]{2}{*}{$p^{1}$} \\
\hline & $n(23)$ & $42,6 \%$ & $n(25)$ & $46,3 \%$ & $\mathrm{n}(6)$ & $11,1 \%$ & $n(54)$ & $100 \%$ & \\
\hline Insônia & 12 & 52,6 & 12 & 48,0 & 3 & 50,0 & 27 & 50,0 & 0,9590 \\
\hline Perda visual & 1 & 4,6 & 2 & 8,0 & - & - & 3 & 5,6 & 0,7040 \\
\hline Labirintite & 2 & 8,7 & 2 & 8,0 & 1 & 16,7 & 5 & 9,3 & 0,7990 \\
\hline HAS & 13 & 56,5 & 20 & 80,0 & 3 & 50,0 & 36 & 66,7 & 0,1480 \\
\hline Doenças Cardiovasculares & 9 & 39,1 & 12 & 48,0 & 2 & 33,3 & 23 & 42,6 & 0,7330 \\
\hline Doença de Chagas & 6 & 26,1 & 5 & 20,0 & - & - & 11 & 20,4 & 0,3680 \\
\hline Depressão & 4 & 17,4 & 8 & 32,0 & 1 & 16,7 & 13 & 24,1 & 0,4490 \\
\hline Diabetes & 4 & 17,4 & 4 & 16,0 & 3 & 50,0 & 11 & 20,4 & 0,1600 \\
\hline Artrose & 11 & 47,4 & 17 & 68,0 & 3 & 50,0 & 31 & 57,4 & 0,3420 \\
\hline Osteoporose & 5 & 21,7 & 7 & 28,0 & 2 & 33,3 & 15 & 25,9 & 0,8040 \\
\hline Hérnia Disco & 6 & 26,1 & 4 & 16,0 & 1 & 16,7 & 11 & 20,4 & 0,6670 \\
\hline
\end{tabular}

GF: grupo frágil; GP: grupo pré-frágil; GN: grupo não frágil; HAS: hipertensão arterial sistêmica.

Os dados estão expressos em porcentagem (\%); $p^{3}$ : Diferenças testadas pelo teste de Qui-Quadrado. 


\section{Discussão}

O presente estudo demonstrou menor percentual de idosos classificados como frágeis e um número expressivo de pré-frágeis, resultados semelhantes a outros estudos nacionais ${ }^{4,11}$ e internacionais. ${ }^{2,12}$ Esse achado ressalta a importância da intervenção em idosos pré-frágeis visto que o maior potencial para prevenção encontra-se neste estágio de transição que antecede as manifestações clínicas da condição de fragilidade. ${ }^{13}$

De forma geral, houve o predomínio do sexo feminino na amostra estudada. Esse achado está de acordo com outros estudos que demonstram a feminilização da velhice ${ }^{14}$. Ao contrário da presente investigação, estudo da rede FIBRA ${ }^{4}$ demonstrou estatisticamente o predomínio de mulheres no grupo frágil e de maior número de homens no grupo não frágil. É reportado na literatura que mulheres apresentam perdas acentuadas das reservas fisiológicas comparadas aos homens ${ }^{15}$, além de estarem mais predispostas a condições socioeconômicas e de saúde desfavoráveis, aspectos que podem refletir em maiores chances de fragilidade. ${ }^{16}$

A faixa etária mais expressiva foi entre 65 e 75 anos, sendo o grupo não frágil seguido pelo frágil os que apresentaram maior percentual de indivíduos, enquanto que nos idosos acima de 75 anos, destacou-se o grupo pré-frágil, apesar da ausência de significância estatística. Estudo conduzido em sete cidades brasileiras ${ }^{4}$ revelou percentuais elevados de idosos acima 75 anos na condição de pré-fragilidade. As características próprias do processo de envelhecimento, o que inclui perdas em todos os sistemas do corpo tanto em aspectos estruturais como funcionais, podem explicar a associação entre fragilidade e idade avançada. ${ }^{17}$

Os estudos ${ }^{4,18}$ demonstram relação entre fragilidade, ausência de escolaridade e baixa renda, ao contrário da presente pesquisa que não evidenciou associação entre essas variáveis. Apesar disso, destaca-se que o analfabetismo foi percentualmente maior entre os frágeis e a categoria 1 e 4 anos de estudo e renda de até 1 salário foi a mais frequente no GP. Acredita-se que essas características possam ser reflexo de desvantagens acumuladas ao longo do curso de vida, somadas às perdas decorrentes do processo de envelhecimento. ${ }^{4}$

Sobre o arranjo de moradia, nos grupos frágil e pré-frágil houve maior percentual de idosos que referiram residir com os filhos, características essas que podem indicar a necessidade de maiores cuidados por parte da família destes idosos, justificando esta distribuição. Quanto ao estado civil, o grupo frágil foi predominantemente viúvo, condizente com estudo nacional. ${ }^{4}$ Já nos demais grupos houve predomínio do estado casado/vive com o companheiro. Possivelmente, a perda do companheiro possa ter se constituído como evento estressor, contribuindo para as manifestações advindas desta síndrome. Sugere-se a necessidade de maiores investigações para compreensão da relação entre essas variáveis, visto a ausência de diferenças significativas entre os grupos e o reduzido número de idosos no GF.

O grupo frágil apresentou maior número de morbidades sendo mais comum a HAS, artrose, diabetes, e insônia; enquanto que para o grupo pré-frágil houve predomínio da HAS, doenças cardiovasculares, artrose e insônia; embora sem diferenças estatísticas entre os grupos. A literatura científica demonstra associação entre a fragilidade e doenças, principalmente a doença cardíaca isquêmica, doença pulmonar obstrutiva crônica, hipertensão arterial e artro$\mathrm{se}^{11,17}$, semelhante aos achados no presente estudo. Uma explicação para tal fenômeno sugere que essa contribuição ocorra por meio de níveis mais baixos de atividade, ou mecanismos que afetem algum processo biológico para a manutenção da homeostase. ${ }^{20}$ Em contrapartida, a presença de doenças crônicas é atribuída como marcador de fragilidade e pode colaborar para o desenvolvimento desta condição. ${ }^{21}$

O número de medicamentos consumidos obteve valores estatisticamente significativos entre os grupos frágil, pré-frágil e não frágil, sendo maior para o primeiro grupo. Este resultado pode ser atribuído ao maior número de morbidades entre o grupo frágil, o que consequentemente leva ao maior consumo de medicamentos. ${ }^{20,21}$ Tal achado está de acordo com a literatura atual, que explica a associação da fragilidade com aumento dos marcadores inflamatórios, maior incidência de reações adversas e redução do metabolismo das drogas. ${ }^{22}$

Pela percepção de saúde foi possível observar o predomínio da categoria "regular" por todos os grupos. Quando comparados à saúde de outras pessoas da mesma idade, os grupos pré-frágil e não frágil classificaram, de forma geral, como melhor, já para o frágil houve uma divisão entre igual e melhor. Apesar da ausência significativa, tais resultados não mantêm uma relação esperada entre os grupos de acordo com a literatura que determina uma má percepção de saúde 
especialmente para o grupo frágil. ${ }^{20,21,23}$ Entretanto, outro estudo demonstrou autoavaliações de saúde altamente positivas. ${ }^{24}$ Os autores explicam que a boa autopercepção de saúde, mesmo na presença de indicadores de vulnerabilidade, por meio de mecanismos de autorregulação cognitivo-emocionais, podem até favorecer a adesão a tratamentos de saúde e a hábitos de saúde. ${ }^{25}$

De maneira geral, neste estudo não foram observadas associações da síndrome de fragilidade com as variáveis socioeconômicas, morbidades e autopercepção de saúde; exceto para o consumo de medicamentos que foi significativamente maior no grupo frágil. Tal resultado pode ser atribuído, em parte à reduzida amostra estudada e ao número de idosos do GF. Outra limitação do estudo foi o fato dos participantes serem de uma amostra por conveniência, e que estudos com base populacional (maior amostragem) poderão trazer resultados mais consistentes. Entretanto, esses fatos não desqualificam o estudo, que prima por apresentar um olhar diferenciado para as questões socioeconômicas e de saúde relacionadas à fragilidade.

Em relação às políticas públicas voltadas ao idoso, observam-se recursos para modificação dos fatores socioeconômicos e de saúde envolvidos no processo de fragilidade. A Política Nacional de Saúde da Pessoa Idosa ${ }^{26}$ afirma ser indispensável incluir a condição funcional ao serem formuladas políticas para a saúde da população idosa. Esta política tem como algumas diretrizes a promoção do envelhecimento ativo e saudável, atenção integral e integrada ao idoso, e como estratégias podemos citar a caderneta de saúde da pessoa idosa que é uma ferramenta de identificação de situações de riscos potenciais para a saúde, traz ao profissional de saúde a possibilidade de planejar e organizar ações de prevenção, promoção e recuperação, objetivando a manutenção da capacidade funcional das pessoas assistidas pelas equipes de saúde. Outra estratégia é o fomento ao acesso e uso racional de medicamentos o que é extremamente relevante, visto o maior consumo de medicamentos no grupo frágil como relatado na presente pesquisa.

Tendo em vista que a síndrome de fragilidade é um indicativo de maiores agravos à saúde, hospitalizações e mortalidade ${ }^{2}$, os resultados desta pesquisa apontaram para a necessidade dos serviços e profissionais de saúde estarem atentos às condições socioeconômicas e de saúde dos idosos atendidos, e, além disso, buscarem estratégias para modificação destas condições.

\section{Conclusão}

O presente estudo identificou que o consumo de medicamentos foi estatisticamente maior para os idosos frágeis, indicando a necessidade de atenção especial a essa característica, dado o diferente perfil de consumo apresentado. Além disso, também identificou variáveis socioeconômicas e de saúde mais frequentes em relação às condições de fragilidade $\mathrm{e}$ pré-fragilidade, como baixa escolaridade e renda, predomínio do sexo feminino, maior número de morbidades e percepção de saúde regular. Os achados desta pesquisa ressaltam a discussão das influências dos componentes sociais e de saúde dos idosos, considerando seu reflexo no nível de fragilidade.

\section{Agradecimentos}

À Secretaria de Educação Superior do Ministério da Educação, à UBS Dona Aparecida Conceição Ferreira e à Secretaria Municipal de Uberaba, MG, Brasil.

\section{Referências}

1. Morley JE, Vellas B, van Kan GA, Anker SD, Bauer JM, Bernabei $R$, et al. Frailty consensus: a call to action. J Am Med Dir Assoc. 2013; 14:392-7.

2. Fried LP, Tangen CM, Walston J, Newman AB, Hirsch C, Gottdiener J, et al. Frailty in older adults: evidence for a phenotype. J Gerontol. 2001; 56:146- 56.

3. Neri AL, Yassuda MS, Araújo LF, Eulálio MC, Cabral BE, Siqueira MEC, et al. Metodologia e perfil sociodemográfico, cognitivo e de fragilidade de idosos comunitários de sete cidades brasileiras: estudo fibra. Cad Saúde Pública. 2013; 29:778-92.

4. Rodrigues NO, Neri AL. Vulnerabilidade social, individual e programática em idosos da comunidade: dados do estudo fibra, Campinas, SP, Brasil. Ciênc Saúde Coletiva. 2012; 17:212939.

5. Geib LTC. Determinantes sociais da saúde do idoso. Ciênc Saúde Coletiva. 2012; 17:123-33.

6. Brucki SMD, Nitrini R, Caramelli P, Bertolucci, PHF, Okamoto IH. Sugestões para o uso do mini-exame do estado mental no brasil. Arq Neuropsiquiatr. 2003;61:777-81.

7. Lebrão ML, Duarte YAO. SABE: saúde, bem estar e envelhecimento. O projeto sabe no município de São Paulo: uma abordagem inicial. Brasília (Brasil): Organização Pan Americana de saúde; 2003.

8. Silva SLA, Silva VG, Máximo LS, Dias JMD, Correa Dias R. Comparação entre diferentes pontos de corte na classificação do perfil de fragilidade de idosos comunitários. Rev Bras Geriatr Gerontol. 2011; 5:130-5.

9. Batistone SST, Neri AL, Cupertino APFB. Validade da escala de depressão do Center for Epidemiological Studies entre idosos brasileiros. Rev Saúde Pública. 2007; 41:589-605. 
10. Souza AC, Magalhães LC, Teixeira-Salmela LF. Adaptação transcultural e análise das propriedades psicométricas da versão brasileira do Perfil de Atividade Humana. Cad Saúde Pública. 2006; 22:2623-36.

11. Amaral FLJS, Guerra RO, Nascimento AFF, Maciel ACC. Apoio social e síndrome da fragilidade em idosos residentes na comunidade. Ciênc Saúde Coletiva. 2013; 18:1835-46.

12. Freiheit EA, Hogan DB, Strain LA, Schmaltz, Patten SP, Eliasziw $\mathrm{M}$, et al. Operationalizing frailty among older residents of assisted living facilities. BMC Geriatr. 2011;11:23.

13. Ferrucci L, Guralnik JM, Studenski S, Fried LP, Cutler GB, Waltson JD. Designing randomized, controlled trials aimed at preventing or delaying functional decline and disability in frail, older persons: a consensus report. J Am Soc Geriatr. 2004; 52:625-34.

14. Bandeen-Roche K, Xue QL, Ferrucci L, Waltson J, Guralnik $\mathrm{JM}$, Chaves P, et al. Phenotype of frailty: characterization in the women's health and aging studies. J Gerontol. 2006; 61:262-6.

15. Chen CY, Wu SC, Chen LJ, Lue BH. The prevalence of subjetive frailty and factors associated with frailty in Taiwan. Arch Gerontol Geriatr. 2010; 50:S43-7.

16. Alvarado BE, Zunzunegui MV, Béland F, Bamvita JM. Life course social and health conditions linked to frailty in latin american older men and women. J Gerontol. 2008; 63:1399406.

17. Avila-Funes JA, Helmer C, Amieva $H$, Barberger-Gateau $P$, Le Goff M, Ritchie K, et al. Frailty among community-dwelling elderly people in france: the three-city study. J Gerontol. 2009; 63:1089-96.
18. Espinoza SE, Jung I, Hazuda H. Lower frailty incidence among mexican american than among european american older adults: the San Antonio longitudinal study of aging. J Am Soc Geriatr , 2010; 58:2142-8.

19. Camarano AA. Envelhecimento da população brasileira: uma contribuição demográfica. In: Freitas EV, Cançado FAX, Gorzoni ML, Organizadores. Tratado de Geriatria e Gerontologia. $2^{\underline{a}}$ ed. Rio de Janeiro: Guanabara Koogan; 2006.

20. Fried LP, Ferrucci L, Darer J, Williamson JD, Anderson G. Untangling the concepts of disability, frailty, and comorbidity: implications for improved targeting and care. J Gerontol. 2004; 59:255-263.

21. Sousa AC, Dias RC, Maciel AC, Guerra RO. Frailty syndrome and associated factors in community-dwelling elderly in northeast Brazil. Arch Gerontol Geriatr. 2012; 54: 95-e101.

22. Li H, Manwani B, Leng SX. Frailty, inflammation, and immunity. Aging and Disease, 2011; 2:466-73.

23. Oliveira DCD, Neri AL, D'Elboux MJ. Variables related to the anticipated support for care in community-dwelling older adults. Rev Latinoam Enferm. 2013; 21:742-9.

24. De Vitta A, Neri AL, Padovani CR. Saúde percebida em homens e mulheres sedentários e ativos, adultos jovens e idosos. Salusvita. 2006; 25:23-34.

25. Liang J, Shaw BA, Krause N, Bennett JM, obayashi E, Fukaya $\mathrm{T}$, et al. How does self-assessed health change with age? A study of older adults in Japan. J Gerontol. 2005; 60:224-32.

26. BRASIL. Ministério da Saúde. Portaria GM no 2.528, de 19 out. 2006. Aprova a Política Nacional de Saúde da Pessoa Idosa. Diário Oficial da União, Poder Executivo, 20 out. 2006. 